\title{
Marie-Jeanne Bérère, théologienne catholique, et la question des femmes dans l'Église
}

Mathilde Dubesset, Donna Singles, Renée Dufourt et Michelle Martin-Grünenwald

\section{(2) OpenEdition \\ Journals}

Édition électronique

URL : https://journals.openedition.org/clio/97

DOI : $10.4000 /$ clio.97

ISSN : 1777-5299

Éditeur

Belin

Édition imprimée

Date de publication : 1 avril 2002

Pagination : 199-207

ISBN : 2-85816-620-X

ISSN : $1252-7017$

Référence électronique

Mathilde Dubesset, Donna Singles, Renée Dufourt et Michelle Martin-Grünenwald, « Marie-Jeanne Bérère, théologienne catholique, et la question des femmes dans l'Église », Clio. Histoire, femmes et sociétés [En ligne], 15 | 2002, mis en ligne le 08 février 2005, consulté le 29 avril 2022. URL : http:// journals.openedition.org/clio/97 ; DOl : https://doi.org/10.4000/clio.97 


\title{
Témoignage
}

\section{Marie-Jeanne Bérère, théologienne catholique, et la question des femmes dans l'Église}

\author{
Entretiens réalisés par Mathilde Dubesset \\ avec Marie-Jeanne Bérère (1995) \\ avec Donna Singles, Renée Dufourt et Michelle Martin-Grünenwald
} (2002)

J'ai rencontré Marie-Jeanne Bérère à Lyon en 1994. C'était une petite femme vive, à la parole claire et précise, avec un bel accent bourguignon. Elle avait accepté une interview, à son domicile lyonnais, le 23 mars 1995. Je souhaitais la revoir pour ce numéro de CLIO, Histoire, Femmes et Sociétés sur les Chrétiennes mais elle est décédée dans l'été 2000 , à 73 ans, sans avoir eu le temps de rédiger ses mémoires. Son itinéraire de femme engagée dans l'Église catholique est celui d'une chrétienne très attachée, dès sa jeunesse, au service de l'Église. Dans les années 1950-1960, cette laique célibataire a travaillé, sans statut, sans reconnaissance sociale, acceptant une situation qu'elle a, par la suite, questionnée et remise en cause en entamant des études à la faculté de théologie de Lyon où elle a mené ses propres recherches et assuré un enseignement dans les années 19701980.

Pour retracer l'itinéraire de Marie-Jeanne Bérère, j'ai sollicité le témoignage de trois de ses collègues et amies : deux théologiennes, Donna Singles, religieuse américaine et Michelle Martin-Grünenwald, laïque, mère de deux enfants et une philosophe, Renée Dufourt, professeure en retraite, «militante féministe engagée au plan politique et religieux". Cette dernière, parmi ses multiples activités, a travaillé de 1989 à 1994 au secrétariat d'État aux droits des femmes et a été membre du Comité national d'éthique.

Le texte qui suit inclut tout d'abord des éléments recueillis en 1995, lors de l'interview de Marie-Jeanne Bérère, sur son enfance et sa formation, puis il 
reprend l'entretien réalisé à Lyon, en janvier 2002, qui a porté essentiellement sur Marie-Jeanne Bérère avec un prolongement sur la question du statut des femmes dans l'Église catholique aujourd'hui.

Née en 1923 dans un bourg de Saône et Loire, d'une famille modeste - le père était mécanicien automobile et la mère s'occupait des quatre enfants -, Marie-Jeanne Bérère a grandi dans un univers où la pratique religieuse faisait partie du quotidien.

Aller au catéchisme était naturel ; à l'école, il y avait peut-être deux filles qui n'étaient pas baptisées, elles étaient connues. Le jeudi matin, c'était la messe des catéchismes, l'après-midi, le patronage. Le dimanche, il y avait la messe et les vêpres. Les enfants avaient leurs bancs, avec au bout des rangées des demoiselles qui vérifiaient si tout le monde était là. Si on allait passer le dimanche dans un autre village, on le disait au curé. Quand mon frère aîné a tout envoyé promener à l'adolescence, ce fut le drame dans la famille.

Bonne élève, elle passe son certificat d'études. Son institutrice l'encourage à continuer au cours complémentaire et elle réussit son brevet en 1939. Son père est favorable à ses projets d'études, sa mère la voit bien demoiselle des postes ou institutrice. Elle se présente en 1940 au concours de l'École normale d'institutrices mais c'est la drôle de guerre, la conjoncture est difficile - un frère mobilisé, le père sans travail - et elle échoue de peu. "C'était un vrai drame de ne pas être reçue». Partie enseigner dans une école catholique à Fontainebleau, elle décide de préparer le baccalauréat tout en assurant ses cours, passe la première partie en 1942. Revenue dans sa région, elle enseigne dans un pensionnat à Mâcon, tout en continuant à préparer la deuxième partie du bac qu'elle obtient en 1944. Au lendemain de la guerre, malade "à cause des privations", elle rentre dans sa famille et, ne sachant que faire, s'engage dans la paroisse, assure le catéchisme, encadre un groupe d'Âmes Vaillantes et réalise que "l'aspect formation chrétienne» l'intéresse beaucoup.

En 1950, à 27 ans, elle est envoyée par le vicaire dans une autre paroisse proche pour assister le curé, "un homme assez inculte mais très pieux et très aimé de ses paroissiens». Elle le suivra dans une paroisse de Bresse aux confins du Jura, à la demande de l'évêque et pourvue d'une dispense canonique car elle a moins de 35 ans.

J'ai tout fait : l'entretien, les courses, le ménage, les repas mais aussi le catéchisme, tout ce qu'il faut faire dans une paroisse. J'ai aussi fait le chauffeur - elle a passé son per- 
mis de conduire - et quand il me restait du temps, je lisais des livres de théologie et je donnais un coup de main pour les homélies, le curé en savait moins que moi, il n'aurait pas été admis en ville.

Elle n'a pas de rémunération fixe, ni de statut, mais elle est nourrie, logée, et déclarée à la sécurité sociale. Cette situation, à l'époque, ne la choquait pas disait-elle en 1995 - car "malgré cette vie austère, je trouvais une certaine satisfaction à faire tout cela». L'expérience se termine en 1959, le prêtre qu'elle assistait étant tombé malade. Elle a 36 ans et est alors embauchée comme enseignante dans un petit séminaire rural, près de Buxy en Saône et Loire, où elle travaille jusqu'en 1970, une expérience qu'elle a souvent évoquée par la suite’.

D.S : Sur le petit séminaire, elle avait des choses étonnantes, pittoresques et malheureuses à raconter. C'était des séminaristes entre 13 ans et 18 ans. Le responsable du séminaire avait dit à Marie-Jeanne, le jour même de son arrivée : «Mademoiselle, évidemment, vous allez prendre votre repas dans votre chambre, vous ne mangerez pas avec nous et puis vous n'assisterez pas non plus au conseil des professeurs parce que, vous comprenez, vous êtes une femme; et puis enfin, qu'on ne vous voie pas trop !» Elle racontait comment, pour la Fête-Dieu, elle ne savait jamais où se mettre dans la procession, avec les professeurs ? avec les jeunes? avec les religieuses qui étaient derrière ? Sa situation était extrêmement ambiguë. Combien de fois elle manquait la communion parce que ces petits jeunes déjà en soutane, des petits prêtres en fleur, avaient la possibilité d'aller à l'autel pour recevoir la communion ; ils faisaient une sorte de procession et puis, quand à la fin, Marie-Jeanne s'approchait du banc de communion, le prêtre lui tournait le dos et c'était trop tard, il mettait déjà l'hostie dans le tabernacle. Constamment elle s'est sentie comment dire ... inexistante ! C'était très dur mais à la fin elle a demandé à l'évêque de compléter sa formation en théologie et on lui a donné une petite bourse pour aller à Lyon. Elle s'est inscrite à l'Institut Pastoral d'Études Religieuses pendant deux ans, au début des années 1970. C'est là que je l'ai connue car j'avais déjà un enseignement. J'ai trouvé cette femme exceptionnellement douée. Henri Denis, qui fut vicaire général du diocèse de Lyon et expert du concile Vatican 2, a reconnu ses dons et c'est lui qui l'a poussée à faire des études de théologie. Elle a fait un parcours très rapide avec des

1 Elle a donné un témoignage lors du colloque annuel du Mouvement Jeunes Femmes (d'origine protestante) en 1991, sur le thème "Dieu a-t-il peur des femmes ?" 
notes très élevées parce qu'elle était brillante, reconnue pour la solidité de sa pensée et de sa réflexion. Sa maîtrise a reçu le prix du jury et a été publiée ${ }^{2}$ puis elle a fait sa thèse mais la publication a été tardive et dans une version très simplifiée 3 . Je me suis toujours dit que si Marie-Jeanne avait été un homme, cela aurait été une des figures intéressantes et importantes dans l'Église de France, mais parce que c'était une femme, c'était impossible. Elle était très active, elle organisait des camps d'été pour des séminaristes qui sont devenus prêtres et même évêques. Elle a enseigné jusqu'en 1988, en particulier la mariologie, l'introduction à la théologie et elle a collaboré à plusieurs revues comme Lumière et Vie et La Revue de Droit Canonique. Mais elle devait aussi enseigner dans un collège privé parce qu'elle avait un petit salaire à la faculté de théologie.

M.D : Elle n'a jamais été professeur titulaire à la faculté de théologie?

D.S : Non car dans les facultés de théologie, il y a très peu de personnes qui ont le titre de professeur titulaire. C'est un titre accordé par Rome, un titre canonique qui correspond à une chaire et qui est accordé à ceux qui ont montré une grande érudition, qui ont beaucoup publié. Vous pensez bien que Rome n'est pas intéressée à donner ce titre à un femme !

M.D : Marie-Jeanne Bérère exprimait-elle parfois le regret de ne pas avoir pu accéder à la prêtrise ou à une responsabilité plus importante dans l'Église?

D.S : Au début oui... on en a parlé, on a parlé de diaconat, on a fait un petit groupe de réflexion sur les ministères ordonnés, une question qui est venue sur le tapis très souvent mais je n'ai pas senti chez elle un désir personnel d'être ordonnée prêtre. Elle était, un peu comme moi, motivée par une certaine indignation. Elle se demandait qu'elles étaient les raisons de fond empêchant l'ordination des femmes et comment pouvait-on justifier cette mise à l'écart, qu'elle avait vécue.

R.D : Je me suis souvent demandée si elle-même avait ressenti cette forme d'appel mais je crois surtout que c'était la place totalement méconnue, ignorée parfois même méprisée des femmes dans l'Église qui était sa motivation principale. Parfois je me suis demandée si elle aurait souhaité l'ordination pour ellemême mais elle ne nous l'a jamais dit, jamais. C'était plus son combat pour

2 Marie-Jeanne Bérère, Le jeu de la tradition dans la pratique masculine du ministère apostolique, Cahiers de l'Institut catholique, Ed. Profac, 1980.

3 Marie-Jeanne Bérère, Marie, l'Église, deux figures symbolisant le salut, thèse soutenue en 1987. Version très abrégée : Marie... Tout simplement, Éditions de L'Atelier, 1999. 
changer la situation des femmes et le ministère qui était essentiel. Elle réfléchissait beaucoup sur cette question et contestait la conception très hiérarchisée, très autoritaire du ministère ${ }^{4}$.

M.M.G : Pour autant, je pense que, inconsciemment, elle a été très marquée par l'indifférence et même le mépris et l'humiliation qu'elle a subis dans ce petit séminaire où elle devait être une ombre, où elle n'existait pas. Cela a nourri non pas un ressentiment mais quand même une position critique parce que cela partait d'une expérience vécue, ce n'était pas une position théorique, elle avait beaucoup souffert!

D.S : Et au lieu de sombrer dans le découragement, cela a eu l'effet contraire, cela lui a donné une énergie étonnante.

R.D : Elle ne donnait jamais l'impression d'être accablée, au contraire, elle était toujours dynamique, toujours vaillante avec une forme de gaîté parfois. Elle était capable d'une ironie forte, mettant bien les choses en perspective.

D.S : Ce qui était important pour elle, c'était d'échanger sur les idées, de développer, d'approfondir les idées, c'était une intellectuelle. Combien de fois elle m'a dit : "mais quel sens tu donnes à ce mot Donna" ?

\section{M.D : Pouvez-vous me parler de la thèse de Marie-Jeanne Bérère?}

D.S : Elle était très intéressée par la figure de Marie, un intérêt qui découlait tout logiquement de son intérêt pour la question des femmes en général. Elle voulait débarrasser cette figure de toutes sortes de mythes, d'imageries qui faisaient de Marie une sorte de déesse mère. Ce que j'en retiens surtout, c'est qu'au fil de l'histoire, on voit que l'Église a parlé d'elle-même à travers la figure de Marie. On peut savoir ce qu'était l'Église à telle ou telle époque à partir de l'iconographie de Marie. À la différence du Moyen Âge où les vierges étaient représentées avec l'Enfant Jésus, dans une position de médiatrices entre Dieu et le monde, les vierges du XIX' siècle sont souvent seules comme celle de Lourdes ou de Fatima. Or c'est une époque où l'Église catholique se referme sur elle-même.

R.D : Il me semble qu'il y a une forme de sacralisation de la figure de la Vierge que dénonçait beaucoup Marie-Jeanne, car, du coup, Marie est très loin des femmes. C'est aussi la sacralisation du clerc qui le met complètement à part et qui renvoit les femmes à une position subordonnée.

4 Elle a écrit à plusieurs reprises dans la revue catholique, au ton très critique à l'égard de l'Eglise, Golias, qui est publiée à Lyon. C'est aussi le cas de mes interlocutrices. 
M.D : Pourquoi cette la thèse a-t-elle été publiée dans une version très allégée?

M.M.G : D'abord cela coûte cher de publier une thèse. Marie-Jeanne n'avait pas de communauté religieuse pour la soutenir, elle était relativement âgée et puis ce sujet dérangeait beaucoup.

D.S : Bruno Chenu' a lu sa thèse et il a été enthousiasmé. Mais on lui a dit qu'on ne pouvait pas la publier parce que Marie-Jeanne «n'était pas connue».

M.D : Vous avez publié avec Marie-Jeanne Bérère, il y a 20 ans, un ouvrage intitulé : Et si on ordonnait des femmes... ? ${ }^{6}$ Comment avez-vous travaillé et que peuton dire sur ce sujet aujourd'hui?

D.S : On avait travaillé dans un groupe sur la question du diaconat et écrit dans une petite revue. Il y avait eu en 1976 le grand texte de Rome Inter Insignores expliquant pourquoi les femmes ne pouvaient pas être ordonnées prêtres. Alors nous avons examiné de près ces différents arguments, c'était une approche intellectuelle et non revendicative. Renée a fourni la partie philosophique, moi je m'occupais du choix des douze apôtres et Marie-Jeanne est intervenue sur la question du sacré.

R.D : Vous veniez chez moi, une fois par semaine, on a vraiment travaillé ensemble, ce qui était tout à fait passionnant.

D.S : Mais après ce fut la catastrophe. Nous n'avions pas fourni de titre, nous n'en n'avions pas et nous ne connaissions pas le monde des éditeurs. Ce titre, on l'a toujours regretté car il ne reflétait pas notre démarche critique. Finalement, l'ouvrage a été peu diffusé et vite envoyé au pilon. Dans les librairies catholiques à Lyon, on le mettait tout en bas, cela sentait trop le soufre!

M.D : Ce livre a-t-il suscité des réactions et lesquelles?

D.S : Louvrage est resté pratiquement inconnu, il y a eu quelques comptes rendus, plutôt mitigés. On était accusées d'être des pétroleuses. La journaliste de La Croix nous a reproché d'être à côté de la plaque, j'ai eu l'impression qu'elle n'avait pas lu le livre.

R.D. Mon sentiment, c'est que nous étions vues comme ne comprenant rien à la position des femmes dans l'Église. Notre analyse, nos critiques, qui n'étaient

5 Bruno Chenu, théologien, longtemps directeur de la chronique religieuse de La Croix, qui s'est beaucoup intéressé à la théologie de la libération, à l'œcuménisme et aux théologies féministes.

6 Marie-Jeanne Bérère, Renée Dufourt, Donna Singles, Et si on ordonnait des femmes... ? Le Centurion, 1982. 
pas forcément des revendications, étaient ressenties comme scandaleuses, aberrantes parce que nous ne comprenions pas - nous disait-on - cette place de la féminité comme un élément fondamental dans la structure de l'Église?.

M.D : Que dire aujourd'hui sur la position des femmes dans l'Église catholique?

D.S : D'abord, on peut dire que les femmes font ce qu'elles peuvent faire et cela peut faire bouger les choses car elles deviennent compétentes et progressivement, on ne peut plus se passer d'elles. Peut-être qu'à long terme, il deviendra logique d'intégrer les femmes dans les lieux de décision. Il y a déjà des femmes canonistes. Mais il y a un problème de fond en ce qui concerne le ministère de l'ordre qu'il faut remettre en question. Quand on regarde ses origines, au $\mathrm{III}^{\circ}$ siècle, on découvre que c'est une institution créée par l'Église, pour faire face à une nouvelle situation. Cette fonction a été sacralisée et on a maintenu cette sacralisation. Or je n'aimerais pas du tout voir les femmes introduites dans ce genre d'ordre.

R.D : C'est la position que nous avons partagée. Dans notre réflexion, il y avait la question de la place des femmes mais aussi celle du ministère et nous allions jusqu'à dire que s'il demeurait ainsi, les femmes ne le demanderaient pas.

M.D : Ne rejoignez-vous pas finalement la position de ces fermmes, actives dans l'Église, qui ne revendiquent pas l'accès au ministère ordonné?

D.S : Je trouve que le choix de ces femmes est difficile parce que, être active dans une paroisse, c'est souvent ne pas être reconnue ; il faut beaucoup de discernement.

R.D : Il y a aussi des femmes actives dans les mouvements d'action catholique qui reprennent cette revendication de la féminité pour que leur travail soit reconnu mais, de mon point de vue, c'est un mouvement régressif. Bien sûr, elles s'affirment mais, en disant que les femmes prennent leur place à côté de toute la cléricature, elles reviennent à cette idée de complémentarité qui s'accommode d'une certaine subordination.

M.D : Je reviens à la question du sacré. Pourquoi cette mise à distance, aussi bien dans les travaux de Marie-Jeanne Bérère sur Marie que dans votre critique du minis-

7 On peut considérer que la démarche critique de ces femmes s'inscrit dans la "crise catholique» analysée par Denis Pelletier dans son récent ouvrage : La crise catholique. Religion, société, politique en France (1965-1978), Payot, 2002. 
tère ordonné?

M.M.G : Jésus a mis en avant la sainteté et non pas le sacré qui est un notion de l'Ancien Testament. En fait, tout un courant du judaïsme est repris à partir du III' siècle, il a traversé le temps et il est maintenant repris par les jeunes clercs. On est de nouveau dans une période où ils sont plus séparés que jamais des autres. On remet les vêtements sacerdotaux, le sacré est partout !

D.S : Avec le sacré on est parfaitement en dehors du christianisme. Bien sûr c'est une réalité qui existe dans l'humanité. Les ethnologues, les anthropologues l'ont montré, le sacré est un sentiment profond chez l'être humain mais cela n'a rien à voir avec Jésus ! Le sacré est lié à l'irrationnel, cela existe mais le problème c'est l'instrumentalisation, l'institutionnalisation de cet irrationnel. Marie-Jeanne avait développé justement ce thème de la nécessaire désacralisation du prêtre comme condition d'accès des femmes au ministère.

R.D : Il y a aussi une autre forme de sacralisation, celle de la féminité !

M.D : Le statut des femmes pasteures dans le monde protestant correspond-il mieux à ce que vous aimeriez voir se réaliser dans l'Église catholique?

M.M.G : Les femmes pasteures président effectivement à l'eucharistie, elles animent la communauté, toutes choses que, nous les femmes, nous pourrions faire aussi dans nos communautés catholiques. Je trouve que ce sont des figures assez proches de ce que nous pourrions imaginer. J'ai assisté, aux États-Unis, à une messe présidée par une femme, dans le rite épiscopalien (anglican) ; j’ai trouvé ça très beau, il y avait beaucoup de respect. Pour une fois c'était une femme qui présidait et qui arrivait à la fin de la procession de sortie comme la personnalité importante.

D.S. : Je ne connais pas bien les pasteures protestantes. Mais l'une d'elles m'a dit que sa position n'est pas si facile dans son Église et que les pasteures sont souvent reléguées dans les tâches secondaires.

M.M.G : Une femme pasteure nous a dit qu'elle souffrair d'une sorte d'ostracisme de la part de certains de ses fidèles mais les choses avancent. Je trouve que la figure de la femme pasteure est assez proche de ce qui pourrait se faire dans l'Église catholique, y compris dans cette dimension désacralisée.

M.D : Les résistances de l'Église catholique, à propos par exemple de l'ordination des femmes, vous ont-elles incitées à la quitter?

M.M.G : Je dirai qu'il faut y être parce que c'est le lieu du combat, la facilité ce serait d'en sortir. Au titre de mon baptême, c'est parce qu'il y a beaucoup de 
difficultés dans l'Église catholique que j'ai l'intention d'y rester.

D.S : Au plan spirituel et religieux, je n'ai pas d'autre maison, c'est aussi simple que ça!

R.D : Comment dire ? Je me souviens avoir dit une fois dans une réunion du groupe Esprit, où on avait eu une discussion sur la foi, que pour moi c'est Dieu qui me résiste. Je dirais finalement que, malgré tout, même si j'ai été tentée d'en sortir et si je me sens parfois plus proche des protestants, eh bien là aussi, c'est la foi qui me résiste. 